\title{
Design of Exponentially Weighted Moving Average Scheme for Standardized Means
}

\author{
A M Razmy ${ }^{1}$ and T S G Peiris ${ }^{2}$ \\ ${ }^{1}$ Department of Mathematical Sciences, Faculty of Applied Sciences, South Eastern \\ University of Sri Lanka, Sri Lanka. \\ ${ }^{2}$ Department of Mathematics, Faculty of Engineering, \\ University of Moratuwa, Sri Lanka. \\ Corresponding Author: amrazmy@seu.ac.lk
}

Received: 23 May 2013 / Revised: 12 November 2013 / Published: 15 November 2013

(C) IAppStat-SL 2013

\begin{abstract}
Exponentially Weighted Moving Average (EWMA) scheme in monitoring process mean is favored because it remembers the past information and detects the small shifts in the mean of a sequence of independent normal variates. However the design procedure of the EWMA scheme was complex till Crowder (1989) presents a simplified scheme for monitoring process mean. Currently standardizing the variables and monitoring them jointly is widely practiced and many schemes are presented under joint quality monitoring. Therefore a need arises to design EWMA schemes for monitoring standardized process means which will easier the work of monitoring more than one process variable at a time. In this paper an alternative design of such a scheme is presented. The new scheme is easy to apply and is independent of sample size. The scheme parameters are the same for any process if the in-control average run length (ARL) and the shifts in means to be detected are the same. In industrial application this property is more beneficial for monitoring few variables in one display.
\end{abstract}

Keywords: Average run length, Exponentially weighted moving average, Process mean, Shift in mean.

\section{Introduction}

In quality control, monitoring of process mean by using Exponentially Weighted Moving Average (EWMA) was introduced by Roberts (1959). Subsequently different procedures of investigating process mean through EWMA were discussed 
by many authors (Robinson and Ho, 1978;, Crowder, 1987;, Chantraine, 1987; and Lucas and Saccicci, 1987). Further a simple design procedure of EWMA scheme for monitoring process mean was reported by Crowder (1989). Chang and Gan (1993) introduced the design procedure of monitoring variance using EWMA. These two design procedures are extensively used in monitoring of process mean and variance simultaneously and these joint monitoring techniques were presented by many authors (Gan, 1995; Chen et al, 2001; and Chen et al 2004).

In industry, EWMA charts are designed for monitoring different process means and these charts are displayed separately. However the quality control engineer should keep on watching all the displays in parallel. As an alternative, use of standardized mean or variance for process monitoring has the advantage of having single chart display for monitoring several process parameters at a time. Further if the average run lengths (ARL) are same for the means to be monitored then the control limits for the chart will also be same. The use of standardized mean and variance and the advantages of this standardization are discussed by McCraken and Chakarborti (2013) with a revision of few joint monitoring schemes with standardized mean and variance. Chen and Cheng (1998) used standardized mean and variance for presenting max chart. The max chart combines two standardized statistics, one for mean and other for variance, by taking the maximum of the absolute values of the two statistics. Razmy (2010) used standardized mean and variance for introducing Shewhart distance scheme for joint quality monitoring. A technique of applying EWMA for standardized variable is useful because a single chart display can be used for monitoring several standardized process variables at a time while remembering the past information to detect the small shifts in the mean of a sequence of independent normal variates. This application can be extended for joint monitoring of standardized process mean and variance in advanced quality control. In this paper, a design of EWMA schmes for standartized mean is proposed with a simple design procedure. This new procedure is illustratred using an application example.

\section{Scheme Development}

Let $X_{t j}$ denote a certain quality characteristic of a process where $t$ is the sample number $(t=1,2, .),$.$j is the j^{\text {th }}$ unit of the sample and $j=1,2, \ldots, n$. It is assumed that $X_{t j}$ 's are independently and identically normally distributed random variables with mean $\mu_{0}$ and standard deviation $\sigma_{0}$. Let 


$$
\bar{X}_{t}=\frac{1}{n} \sum_{j=1}^{n} X_{t j}
$$

be the $t^{\text {th }}$ sample mean and

$$
S_{t}^{2}=\frac{1}{n-1} \sum_{j=1}^{n}\left(X_{t j}-\bar{X}_{t}\right)^{2}
$$

be the $t^{\text {th }}$ sample variance. The sample mean for each sample is standardized as

$$
U_{t}=\frac{\bar{X}_{t}-\mu_{0}}{\sigma_{0} / \sqrt{n}}
$$

and when a process is in-control, $U_{\mathrm{t}}$ is standard normal random variable. The EWMA chart for monitoring the mean based on standardized sample mean is obtained by plotting

$$
Q_{t}=\left(1-\lambda_{M}\right) Q_{t-1}+\lambda_{M} U_{t}
$$

against the sample number where $\lambda_{M}$ is a positive smoothening constant such that $0<\lambda_{M} \leq 1$. $Q_{0}$ is the $E\left(U_{t}\right)$ for the process and it is zero. Variance of $Q_{t}$ is written as

$$
\operatorname{Var}\left(Q_{t}\right)=\frac{\sigma_{0}^{2}}{n}\left(\frac{\lambda_{M}}{2-\lambda}\right)\left[1-(1-\lambda)^{2 t}\right]
$$

as reported in Crowder (1989). An out-of-control signal is issued if $Q_{t}$ is greater than the upper control limit (UCL) $H_{M}$ or $Q_{t}$ is less than the lower control limit (LCL) $-H_{M}$. The control limits for the selected $\lambda_{M}$ values are decided based on ARL that is the average number of samples taken before an out-of-control signal issued when the process is actually in-control. The control limits for selected $\lambda_{M}$ values can be calculated based on the equations

$$
\begin{aligned}
& U C L=\mu_{0}+L \frac{\sigma_{0}}{\sqrt{n}} \sqrt{\frac{\lambda_{M}}{2-\lambda_{M}}\left[1-\left(1-\lambda_{M}\right)^{2 t}\right]} \\
& L C L=\mu_{0}-L \frac{\sigma_{0}}{\sqrt{n}} \sqrt{\frac{\lambda_{M}}{2-\lambda_{M}}\left[1-\left(1-\lambda_{M}\right)^{2 t}\right]}
\end{aligned}
$$

where $L$ is the number of standard deviation unit that is based on the selected ARLs such that

$$
\frac{1}{A R L}=P\left(L C L>\mu_{0}>U C L\right) \text {. }
$$

The calculated control limits for selected values of $\lambda_{M}$ and ARL are displayed in Figure 1. These calculated control limits were rechecked using simulations. When 
$\lambda_{M}=1$, the value of $Q_{t}$ depends only on the most recent sample $t$, just as the case of the Shewhart $\bar{X}$ bar chart (Shewhart, 1939). The optimal value of $\lambda_{M}$ differs based on the shift in mean to be detected quickly and the ARL of the scheme. The shift in mean is normally said in number of standard deviation units away from the incontrol mean. The new mean after a shift can be written as

$$
\mu=\mu_{0}+\Delta \frac{\sigma_{0}}{\sqrt{n}}
$$

where $\Delta$ is the shift from the in-control mean in number of standard deviation units. To find an optimal $\lambda \mathrm{M}$ for detecting a particular shift in mean quickly for a particular in-control ARL, the control limits for various $\lambda \mathrm{M}$ values $(\lambda \mathrm{M}=0.025$, $0.050, \ldots, 0.095,1.000$ ) were found. Then for a particular shift, the $\lambda \mathrm{M}$ value that gives the smallest out-of-control ARL is the optimal $\lambda \mathrm{M}$ for that particular shift. The optimal $\lambda \mathrm{M}$ for detecting different shifts in mean $(\Delta)$, for various values of ARL were found using simulations and these are displayed in Figure 2. In all cases simulations were run until the standard errors of the ARL's were less than $1 \%$ of the pre-specified ARL's.

\section{Scheme Design}

The design procedure recommended by Crowder (1989) for his EWMA scheme is adopted for the design of this EWMA scheme for the standardized mean. The design strategy is to find $\lambda_{M}$ for a given in-control ARL that minimizes the out-ofcontrol ARL for a specified shift in the mean. To design this scheme, the following steps are recommended:

Step 1. Select the smallest acceptable in-control ARL for the scheme.

Step 2. Determine the magnitude of the shift in mean $(\Delta)$ to be detected quickly. Select the $\lambda_{M}$ that gives the minimum out-of-control ARL at the shift selected.

Step 3. Given the value of $\lambda_{M}$ determined in Step 2, find the control limit $H_{M}$, for the in-control ARL selected in step 1 .

This scheme can then be implemented by plotting the monitoring statistics $Q_{t}$ against the sample number $t$. A signal is issued if $Q_{t}>H_{M}$ or $Q_{t}<-H_{M}$. In Step 2, the value of $\lambda_{M}$ can be read off easily from Figure 2 given the value of $\Delta$ and incontrol ARL. Similarly using the value of $\lambda_{M}$ obtained in Step 2, the control limit $H_{M}$ can be read off from Figure 1 . 


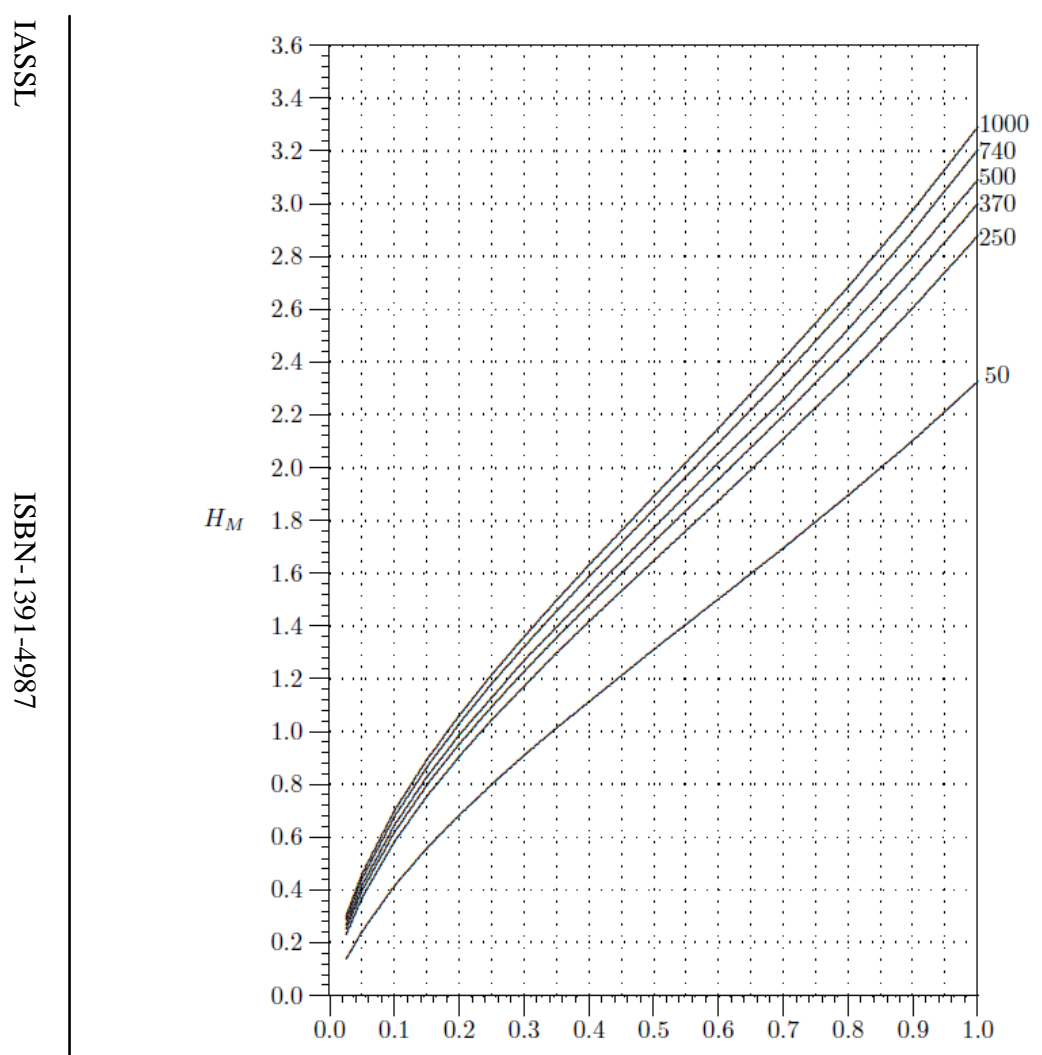

Figure1: Control limits for the EWMA mean scheme based on standardized sample means with in-control ARLs of 50, 250, 370, 500, 740 and 1000 .

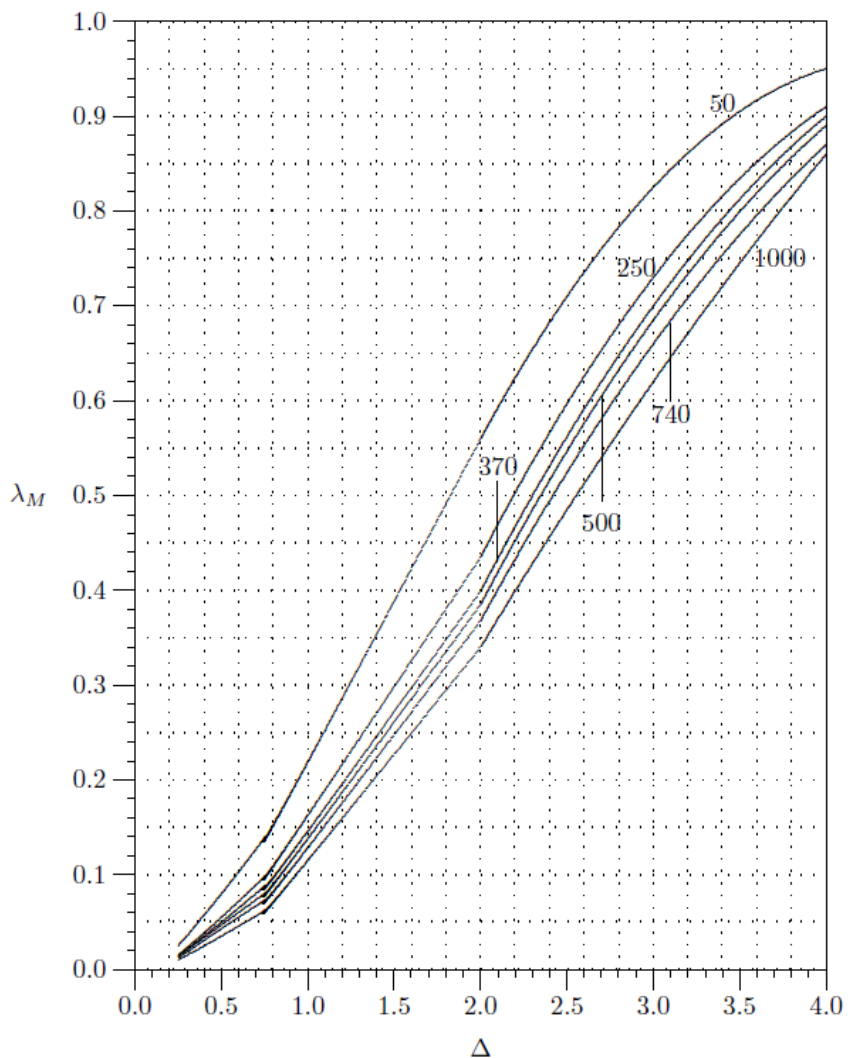

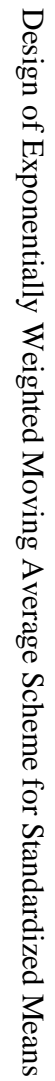

Figure 2: Optimal $\lambda_{M}$ values for detecting various shifts in mean for the EWMA mean scheme based on standardized sample means with in-control ARLs of 50, 250,370, 500, 740 and 1000. 


\section{Application}

If a scheme needs to have a quick detection (minimum out-of-control ARL) when there is one unit standard deviation shift in the mean $(\Delta=1)$ for the in-control ARL of 740 , the following step can be carried out.

Step 1. Set the desired in-control ARL of the scheme that is ARL $=740$.

Step 2. If $\Delta=1$ shift in mean is to be detected quickly, the optimal $\lambda_{M}=$ 0.125. (obtained from Figure 2 for ARL $=740$ and $\Delta=1$ ).

Step 3. From Figure 1, the control limit $H_{M}$ is 0.66 , based on $\lambda_{M}=0.125$ and ARL $=740$.

Then the EWMA scheme for standardized means can be implemented by plotting the monitoring statistics $\mathrm{Q}_{t}$ against the sample number where a signal is issued if

$$
-0.66>Q_{t}>0.66 \text {. }
$$

\section{Conclusion}

This paper provides an easy way of designing EWMA scheme for the standardized means. As means are standardized, in industrial application, several parameters can be monitored simultaneously in a single display. A big advantage of these schemes is that the scheme parameters are independent of the sample size which gives more freedom to the quality engineers in selecting sample size. Another advantage of these schemes is that the scheme parameters are the same for any process if the incontrol ARL and the shifts to be detected are the same. For example the scheme parameters for a process with $\mathrm{N}(25,3)$ with sample size 5 and for a process with $\mathrm{N}(200,10)$ with the sample size 10 are the same if the in-control ARL and shifts in mean to be detected are the same. This design procedure will be helpful in developing combined monitoring schemes of parameters which is being a popular researchable area in quality control.

\section{Acknowledgment}

This work was supported by the South Eastern University of Sri Lanka and the Higher Education for the Twenty First Century Project, Sri Lanka [SEUSL/OAS/N1]. 


\section{References}

1. Chang, T. C. and Gan, F. F. (1993) - Optimal designs of one-sided EWMA charts for monitoring a process variance. Journal of Statistical Computing \& Simulations, 49, pp 33-48. http://dx.doi.org/10.1080/00949659408811559

2. Chantraine, P. M. R. (1987) - Geometric moving average charts in a manufacturing environment. Paper presented at the 31st annual full technical fall technical conference, Atlantic, NJ.

3. Chen, G. and Cheng, S. W. (1998) - Max chart: Combining X-Bar chart and Schart. Statistica Sinica, 8, pp 263-271.

4. Chen, G., Cheng, S. W. and Xie, H. W. (2001) - Monitoring process mean and variability with one EWMA chart. Journal of Quality Technology, 33, pp 223233.

5. Chen, G., Cheng, S. W. and Xie, H. W. (2004) - A new EWMA control chart for monitoring both location and dispersion. Quality Technology \& Quantitative Management, 1, 217-231.

6. Crowder, S. V. (1987) - A simple method for studyingrun length distributions of exponentially weighted moving average charts. Technometrics, 29, pp 401407. http://dx.doi.org/10.2307/1269450

7. Crowder, S. V. (1989) - Design of exponentially weighted moving average schemes. Journal of Quality Technology, 21, pp 155-162.

8. Gan, F. F. (1995) - Joint Monitoring of process mean and variance using exponentially weighted moving average control charts. Technometrics, 37, pp 446-453. http://dx.doi.org/10.1080/00401706.1995.10484377

9. Lucas, J.M. and Saccucci, M.S. (1987) - Exponentially weighted moving average control schemes: Properties and enhancements. Drexel University Faculty working series paper, 87 pp 5.

10. McCraken, A.K. and Chakarborti, S. (2013) - Control charts for joint monitoring of mean and variance: An overview. Quality Technology \& Quantitative Management, 10, pp 17-36.

11. Razmy, A.M. (2010), Joint monitoring of process mean and variance with shewhart distance scheme, Sri Lankan Journal of Applied Statistics, 11, pp 1426. 
12. Roberts, S.W. (1959) - Control chart based on geometric moving averages. Technometrics, 1 , pp

239-250. http://dx.doi.org/10.1080/00401706.1959.10489860

13. Robinson, P.B. and HO, T.Y. (1978) - Average run lengths of geometric moving average charts by numerical methods, Technometrics, 20, pp 8593.http://dx.doi.org/10.1080/00401706.1978.10489620

14. Shewhart, W. A. (1939) - Statistical methods from the viewpoint of quality control, Washington D.C, Graduate School, Department of Agriculture. pp 75 